\title{
COVID-19 Diagnosed by Serological Antibody Test: A Case Report
}

\author{
Mei Zhou ${ }^{1}$, Xianzhi Xiong ${ }^{1, *}$
}

\author{
${ }^{1}$ Department of Respiratory and Critical \\ Care Medicine, Union Hospital, Tongji \\ Medical College, Huazhong University of \\ Science and Technology, Wuhan, \\ P. R. China \\ *Correspondence \\ xxz0508@hust.edu.cn \\ (Xianzhi Xiong)
}

\begin{abstract}
The new coronavirus, severe acute respiratory syndrome coronavirus 2 (SARS-CoV-2), first reported in Wuhan, China, is causing a pandemic. With an increased understanding of the disease, the diagnosis and treatment guidelines are being updated and improved. In addition to nucleic acid detection, antibody detection kits are also being developed and approved. A medical worker suspected of having COVID-19 in our hospital had fatigue and loss of appetite and pulmonary infection indicated by $\mathrm{CT}$, but the nucleic acid test was negative three times. Finally, she was confirmed to have COVID-19 by a serological antibody test. After rest and oral antiviral treatment, she recovered and returned to work. This is a case report that focuses on suspected mild patients who tested negative for nucleic acids. Such a group of patients had to choose home isolation treatment during the outbreak. The majority of them did not make a definitive diagnosis or rule out COVID-19 by the time they recovered. The antibody test is of great significance to identify infected patients with multiple negative nucleic acid tests. It can complement nucleic acid testing to enhance diagnostic efficiency. We have reviewed the literature and realized that further validation and standardization of serological tests are needed for more mature application of antibody tests to clinical diagnosis and vaccine development.
\end{abstract}

\section{Keywords}

COVID-19, Serological antibody test, Case report

\section{Introduction}

Since December 2019, several cases of unexplained pneumonia have been found in some hospitals in Wuhan, Hubei Province, China. The disease has been confirmed to be an acute respiratory transmission disease caused by SARS-CoV2 [1]. With the rapid spread of the virus, as of August 3, 2020 , more than 80,000 people have been diagnosed with COVID-19 in China, and more than 18,000,000 people have been diagnosed abroad [2]. On March 11, the WHO characterized COVID-19 as a pandemic [3]. At the beginning of the epidemic, the diagnosis was based on RT-PCR detection of SARS-CoV-2 nucleic acid or viral gene sequencing showing sequences that are highly homologous with known SARS$\mathrm{CoV}-2$. However, the accuracy of nucleic acid detection is limited by the viral load and sampling. Many suspected patients have multiple negative nucleic acid tests, which facilitates their role as a potential source of infection and complicates the prevention and control of the epidemic situation. It is urgent to find more convenient and accurate detection methods to assist in diagnosis. Therefore, SARS-CoV-2 antibody testing kit has been developed and approved, and positive results have been included as one of the diagnostic criteria in the Chinese guidelines for the diagnosis and treatment of COVID-19 [4]. A nucleic acid-negative patient in our department diagnosed by serological antibody test is reported as follows. Unlike other reported COVID-19 cases, this is a physician's experience of self-observation and self-treatment during home isolation. Nucleic acid and antibody tests were performed repeatedly during the 2-month observation period.

\section{Case Presentation}

The patient is a 34-year-old female physician. On the evening of January 17, 2020, she felt weak after work and had mild throat discomfort. She had been in good health, and her colleagues and family members had no similar symptoms. The next day, she worked half of a day, as usual, in the morning. She took cold medicine, but there was no noticeable relief from her fatigue. Physical examination revealed no obvious abnormalities. In the afternoon, she performed a routine blood test and five respiratory pathogen antibody tests. The results showed that her lymphocytes were slightly low $(1.01 \mathrm{G} / \mathrm{L})$, and the antibodies against the five common respiratory pathogens (mycoplasma, chlamydia, adenovirus, respiratory syncytial virus, and coxsackie virus) were negative. On the morning of January 19, 2020, she did not feel better but still felt asthenia and loss of appetite. CT examination of the lung showed flakelike ground glass opacity shadows in the dorsal segment of the right upper lobe (Fig. 1A). A nasopharynx swab was used to 

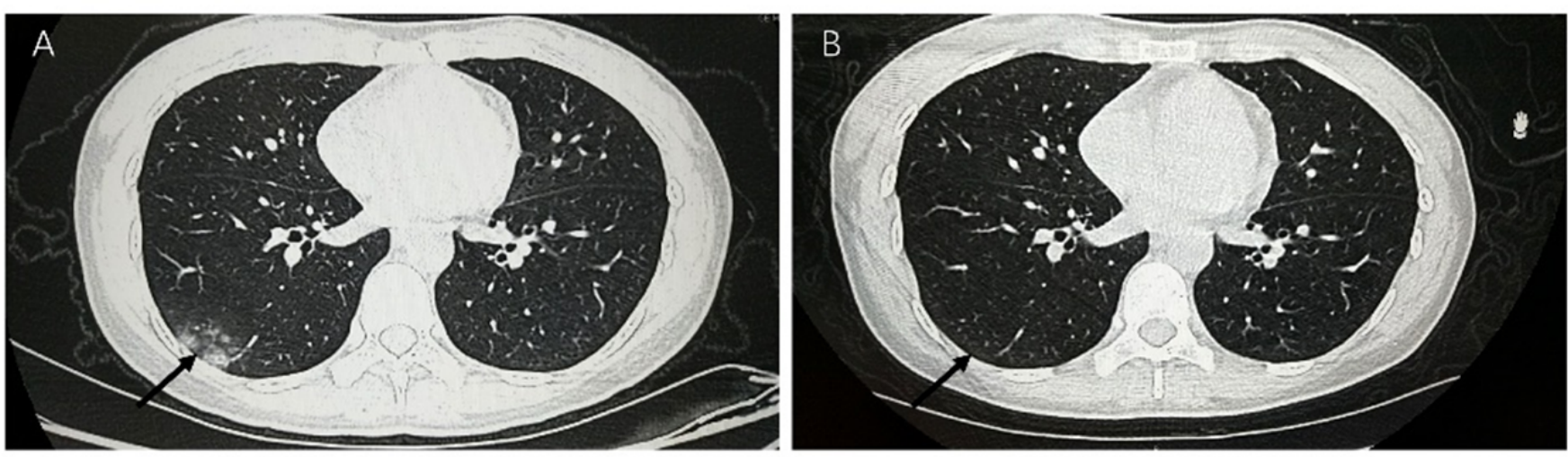

F I G U R E 1. Comparison of lung CT scans.

Lung CT scan on January 19 (illness day 3). (B) Lung CT scan on January 31 (illness day 15).

detect the RNA of influenza A, influenza B and respiratory syncytial virus, and the results were negative. As of that time, there were no positive etiology results. She is a respiratory physician who had been to the isolation ward several times recently. Combined with her epidemiological history and pulmonary imaging changes, by ruling out other common respiratory virus infections, we considered the possibility of COVID-19. Considering the patient's mild symptoms and absence of fever, home isolation and empirical oral medication were recommended. Treatment with oseltamivir $(75 \mathrm{mg})$ twice per day and moxifloxacin $(0.4 \mathrm{~g})$ once per day was performed. Two days later, she could not tolerate gastrointestinal symptoms (nausea and vomiting), so we changed moxifloxacin to levofloxacin (Fig. 2).

On the night of January 21 (illness day 5), an oral swab was taken for a SARS-CoV-2 nucleic acid test, and the result was negative. At the same time, routine blood examination showed a normal range of lymphocyte and C-reactive protein (CRP) levels. Considering that the lymphocyte level returned to normal and there was no obvious evidence of bacterial infection, she discontinued antibiotic therapy. On January 23 (illness day 7), oseltamivir was replaced with arbidol (200 mg, three times a day) because arbidol became available from the pharmacy. After 5 days of oral administration of arbidol, the symptoms of asthenia were significantly improved, and the drug was discontinued on January 28 (Fig. 2). On the afternoon of January 30 , she felt weak and lost her appetite again. The symptoms were similar to those at the beginning of the disease. She started taking arbidol again. On January 31 (illness day 15), a CT scan showed that most of the ground glass shadow in the dorsal segment of the right lower lobe was absorbed (Fig. 1B), and routine blood tests and CRP levels were normal.

After another week of oral administration of arbidol, the symptoms were significantly improved. She had only a minor dry cough, without obvious fatigue or poor appetite. On February 11 (illness day 26), a SARS-CoV-2 nucleic acid test of a throat swab was still negative. At the same time, she was informed that a SARS-CoV-2 serological antibody test was being carried out in our hospital for research purposes[5]. A peripheral blood sample was collected for serological antibody testing, and the results showed that IgM antibody (S/CO: 2.5) and IgG antibody (S/CO: 5.6) were both positive (ELISA kits,
Livzon Inc, Zhuhai, P.R.China, lot number of IgM: 20200308, IgG: 20200308) (Fig. 2). At that point, she was definitely diagnosed with COVID-19. Fortunately, before that, she had made a full recovery as a suspected patient. A month later, her IgM became negative, and her IgG remained positive. Her nucleic acid test was still negative (Fig. 2). To date, none of her family members have developed any suspected symptoms, and close contacts (her husband) tested negative for antibodies and nucleic acids.

\section{Discussion}

At the beginning of the outbreak, due to the rapid increase in the number of patients and limited testing methods, it was impossible to test all suspected patients and clearly diagnose them. Now, as the number of patients declines and the tests become more varied in China, we can test all suspected patients and routinely screen all hospitalized patients and their families in high-risk areas. Although antibody detection is more convenient and faster than nucleic acid testing, it is currently impossible to replace nucleic acid detection. RT-PCR-based viral RNA detection is sensitive and can effectively confirm early SARS-CoV2 infection [6]. Antibody detection may play an important role in the diagnosis of COVID-19 as complement approach in the middle and later stage [5]. Ya-ting $\mathrm{N}$ et al. discussed how to interpret the results of combined detection [7].

The study of Zhang YC et al. reported that seroconversion was observed in $100 \%$ (17/17) of symptomatic patients during the observation period. Nevertheless, only one person $(1 / 5)$ with humoral responses among asymptomatic carriers [8]. Zhang $\mathrm{W}$ et al. found that antibody detection has a high positive rate on 5 days after admission, which was normally considered as a transition from earlier to later period of infection [9]. This is consistent with the result from our department [5]. Quan-Xin Long et al. reported that the median day of seroconversion for both IgG and IgM was 13 days post symptom onset. Seroconversion for IgG and IgM occurred simultaneously or sequentially. Both IgG and IgM titers plateaued within 6 days after seroconversion [10].

The spike and nucleocapsid proteins are the main immunogens of the SARS-CoV-2 [11]. The sensitivity and specificity 


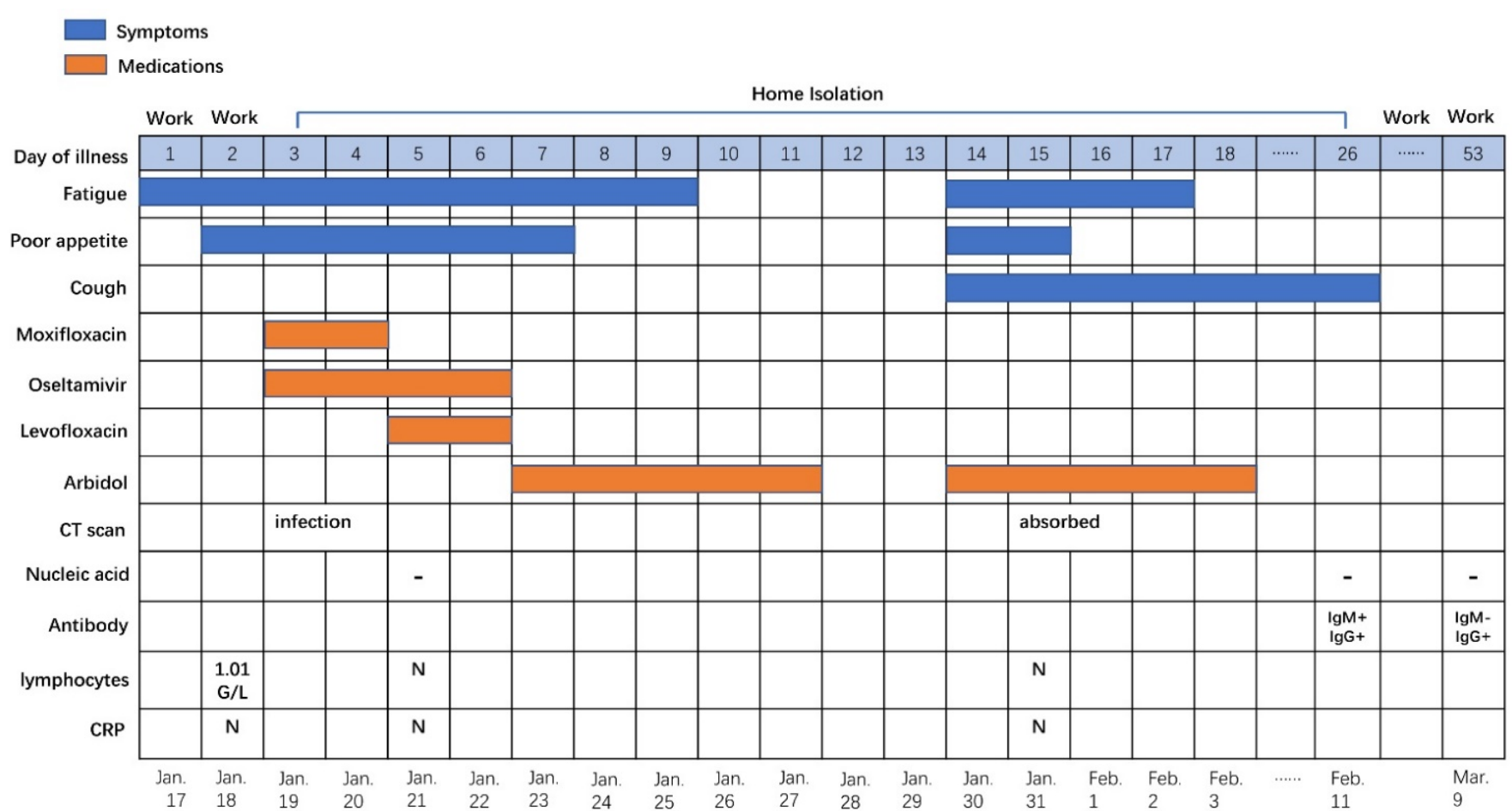

F I G U R E 2. Timeline of disease course according to days from initial presentation of illness and days from home isolation, from Jan 17-Mar 9, 2020.

Abbreviations: $N$, normal; CRP, C-reactive protein.

of ELISA assays for different antigens were different [12]. Moreover, no studies have addressed cross-reactivity from other non-specific proteins that can lead to false-positive result. Therefore, further studies and optimization of ELISA assays will be necessary. Well-defined standard references should be developed to standardize the serologic assays developed by different laboratories. Finally, validation and standardization of serologic testing are important for evaluating vaccine immunogenicity and effective induction of antibody responses for prevention [13].

Our case report has limitations. First, since the SARSCoV-2 serological antibody test kit needs time for development and approval, we have not been able to dynamically monitor serological antibodies in the early stage of this case. Second, in this case, symptoms were obviously relieved, and the CT imaging changes were also significantly resolved by only taking arbidol. It is still unknown whether arbidol is effective against SARS-CoV-2 or whether patients with mild and common infections are likely to self-cure in a certain period of time. Some antiviral drugs, such as lopinavir/ritonavir, arbidol and remdesivir, may be effective against SARS-CoV-2, as indicated in some studies and case reports [14, 15].

In conclusion, antibody testing can help confirm the diagnosis of suspected patients with negative nucleic acid testing, and the combined application can improve the detection efficiency of COVID-19. Furthermore, validation and standardization of serologic testing are necessary for better application of antibody tests to clinical diagnosis and vaccine design.

\section{ACKNOWLEDGEMENTS}

The authors thank Dr. Peng-Cheng Cai at Department of Clinical Laboratory, Union Hospital, Tongji Medical College, Huazhong University of Science and Technology for offering free antibody tests in the early outbreak.

\section{CONFLICTS OF INTEREST}

The authors declare that there is no conflicts of interest for their options and suggestions.

\section{ETHICAL STATEMENT}

The authors are accountable for all aspects of the work in ensuring that questions related to the accuracy or integrity of any part of the work are appropriately investigated and resolved. Written informed censent was obtained from the patient for publication of this study and any accompanying images. A copy of written censent is available for review by the Editor-in-Chief of this journal.

\section{REFERENCES}

[1] Huang C, Wang Y, Li X, et al. Clinical features of patients infected with 2019 novel coronavirus in Wuhan, China. The Lancet. 2020;395:497-506.

[2] Live update: new coronavirus pneumonia outbreak map. 2020. accessable on: https://voice.baidu.com/act/newpneumonia/ newpneumonia/?from=osari_pc_3. (accessed 2020/8/3)

[3] Coronavirus (COVID-19) events as they happen. 2020. accessable on: https://www.who.int/emergencies/diseases/novel- 
coronavirus-2019/events-as-they-happen.

(accessed 2020/3/13)

[4] The Central People's Government of the People's Republic of China. 2020. accessable on: http://www.gov.cn/ zhengce/zhengceku/2020-03/04/5486705/files/ ae61004f930d47598711a0d4cbf874a9.pdf. (accessed 2020/3/13)

[5] Xiang F, Wang X, He X, et al. Antibody Detection and Dynamic Characteristics in Patients with COVID-19. CLIN INFECT DIS. 2020.

[6] Zou L, Ruan F, Huang M, et al. SARS-CoV-2 Viral Load in Upper Respiratory Specimens of Infected Patients. N Engl J Med. 2020;382:11771179.

[7] Ning Y, Hou X, Lu W, et al. Application of the Technology of Serum Specific Antibody in Detection COVID-19. Medical Journal of Peking Union Medical College Hospital. 2020;1-9.

[8] Yongchen Z, Shen H, Wang X, et al. Different longitudinal patterns of nucleic acid and serology testing results based on disease severity of COVID-19 patients. EMERG MICROBES INFEC. 2020;9:833-836.

[9] Zhang W, Du R, Li B, et al. Molecular and serological investigation of 2019-nCoV infected patients: implication of multiple shedding routes. EMERG MICROBES INFEC. 2020;9:386-389.
[10] Long Q, Liu B, Deng H, et al. Antibody responses to SARS-CoV-2 in patients with COVID-19. NAT MED. 2020;26:845-848.

[11] Meyer B, Drosten C, Muller MA. Serological assays for emerging coronaviruses: challenges and pitfalls. VIRUS RES. 2014;194:175-183.

[12] Okba N, Muller MA, Li W, et al. Severe Acute Respiratory Syndrome Coronavirus 2-Specific Antibody Responses in Coronavirus Disease Patients. EMERG INFECT DIS. 2020;26:1478-1488.

[13] Touma M. COVID-19: molecular diagnostics overview. Journal of molecular medicine (Berlin, Germany). 2020;98:947-954.

[14] $\mathrm{Lu} \mathrm{H.} \mathrm{Drug} \mathrm{treatment} \mathrm{options} \mathrm{for} \mathrm{the} \mathrm{2019-new} \mathrm{coronavirus} \mathrm{(2019-}$ nCoV). BIOSCI TRENDS. 2020;14:69-71.

[15] Holshue ML, DeBolt C, Lindquist S, et al. First Case of 2019 Novel Coronavirus in the United States. NEW ENGL J MED. 2020;382:929936.

How to cite this article: Mei Zhou, Xianzhi Xiong. COVID-19 Diagnosed by Serological Antibody Test: A Case Report. Signa Vitae. 2020;16(2):195-198. doi:10.22514/sv.2020.16.0060. 